International Journal of Engineering \& Technology, $7(3)(2018)$ 1735-1739
International Journal of Engineering \& Technology
SPC
Website: www.sciencepubco.com/index.php/IJET
doi: $10.14419 /$ ijet. $v 7 i 3.14122$
Research paper

\title{
Impact of buffer size and TTL on DTN routing protocols in intermittently connected mobile networks
}

\author{
Md. Sharif Hossen ${ }^{1 *}$, Md. Masum Billah², Suraiya Yasmin² \\ ${ }^{1}$ Department of Information and Communication Technology, Comilla University, Bangladesh \\ ${ }^{2}$ Dept. of Computer Science and Information Technology, Bangabandhu Sheikh Minibar Rahman Agricultural University, Bangladesh \\ *Corresponding author E-mail:mshossen@cou.ac.bd
}

\begin{abstract}
Delay-Tolerant Networks (DTNs) are kinds of networks where there does not exist any complete end-to-end route from source to destination. Such networks can also be referred to as Intermittently Connected Mobile Networks (ICMNs), which are featured by asymmetric data rates, large delay, limited resources and high error rates. In this network, size of buffer and Time-to-Live (TTL) for fixed number of nodes and message generation rates contribute to the network performance because of limited resources and short life span of a packet in the net-work. Therefore, investigating efficient routing for altering TTL and size of buffer is very important for overall network performance. This paper presents a performance analysis based on simulation of the impact of buffer size and TTL for several DTN routing protocols in ICMNs scenario. ONE, i.e., Opportunistic Network Environment is used to simulate the routing protocols considering three performance metrics: delivery ratio, mean latency and overhead ratio. Investigated results mention that Spray-and-Focus (SNF) routing exhibits the best performance for altering TTL and size of buffer than other DTN routing protocols, i.e., Epidemic, PRoPHET, PRoPHETv2, MaxProp, RAPID, and Binary-SNW in the considered performance metrics and simulation scenario.
\end{abstract}

Keywords:Delay-Tolerant Networks; Intermittently Connected Mobile Networks; Routing; Routing Protocols; Message Replication; Simulation; Delivery Probability; Average Latency; Overhead Ratio; Opportunistic Network Environment Simulator

\section{Introduction}

Delay-Tolerant Networks (DTNs) are kinds of mobile ad-hoc networks, where there is no persistent route from source to destination. It is an intermittent and sparsely connected mobile ad-hoc network due to fixed transmission range and mobility model. Hence, the network exists in an abrupt change of delay and message errors [1-2]. In such challenging network, popular ad-hoc routing protocols like ad-hoc on-demand distance vector [3] and dynamic source routing [4] cannot be implied for selecting a route to send data properly, as they require an uninterrupted way of communication between two nodes. DTNs are named as Intermittently Connected Mobile Networks (ICMNs) [5] where there exists an intermittent gateway and physically burst connections [6]. It takes benefits using "store and carry" technique to easily reach the messages between two nodes in the network [7-9] as illustrated in Figure 1.

DTNs are mainly seen in the areas of interplanetary networks [10], underwater networks [11], wildlife tracking sensor networks [8], satellite communication [12], vehicular ad-hoc networks [13], and military networks, etc.

In this paper, the several replication-based DTN routing protocols have been analyzed as applied to intermittently connected mobile networks, with focus on Epidemic [14], PRoPHET [15], PRoPHETv2 [16], MaxProp [7], RAPID [17], Binary-Spray-andWait (B-SNW) [18] and Spray-and-Focus (SNF) [19] for varying buffer size and TTL, respectively [20-21].

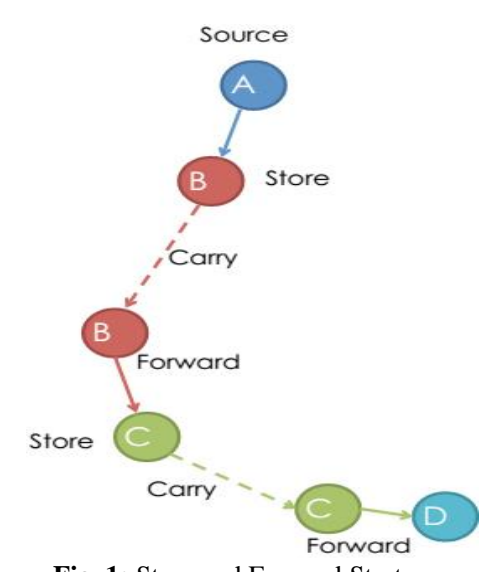

Fig. 1: Store and Forward Strategy.

Although the simulation of these DTN routing protocols is not novel, obtained result in the context is new (added SNF routing) and provides an efficient comparision seen in the simulation. For example, SNF, with specific metrics in a given network scenario could be the best due to the mechanism of the protocol. Studying the results of simulation helps to understand the working comparision of routings to determine what can be achieved in the networks.

The remaining part of the paper is maintained as following: The brief discussion of DTN routings are shown in Section II. The simulation setting and discussion of the simulator is included in Section III. Section IV discusses the obtained results. Overall 
sumurization with future plans about this research endeavor are discussed in Section V.

\section{Routing protocols under investigation}

The considered replication-based DTN routing protocols, namely Epidemic, PRoPHET, PRoPHETv2, MaxProp, Rapid, BinarySNW, and SNF have been discussed in this section.

\subsection{Epidemic}

Epidemic routing [22] is a type of flooding in which a node replicates a message to other when encountered that do not have a message copy in common.

\subsection{Probabilistic routing protocol using history of en- counters and transitivity (PRoPHET)}

PRoPHET routing works like Epidemic but here only distinction is that when two nodes are met, messages are sent to a node that has the higher delivery probability. It includes a transitivity technique (controlled by scaling constant, $\beta$ ) for having a case of rarely meeting two nodes, but there is another node that frequently meets both of these nodes.

\subsection{Probabilistic routing protocol using history of en- counters and transitivity version 2 (PRoPHETv2)}

PRoPHET works well if the value of $\beta$ is zero since it effectively disables the transitivity properties. However, if $\beta>0$, the delivery predictability increases rapidly regardless of encountering any node. This problem can be solved by using PRoPHETv2, another version of PRoPHET, was proposed by Grasic, Davies and Doria.

\subsection{Resource allocation protocol for intentional DTN (RAPID)}

This routing optimizes a single routing metric, i.e., average delay, missed deadlines, or maximum delay to model DTN routing as a utility-driven resource allocation problem. This optimization is achieved by assigning a value to each packet considering the metric. This utility value is the value for which the packet picks good routing. This routing uses those packet first having highest value of utility.

\subsection{Maxprop}

MaxProp routing defines a ranked list to determine which packets should be transmitted first and dropped first. This routing selects the desired shortest path depending on low hop count from source to destination as cost estimate.

\subsection{Spray-and-wait}

Spray-and-Wait (SNW) routing protocol limits the message copies to have good delivery. It has two phases: spray and wait. In spray phase from source, $\mathrm{L}$ copies are forwarded to first $\mathrm{L}$ distinct relays. If these relays are not the destination, then these are in wait phase and attempt to send messages directly to the destination. It has two versions: vanilla and binary. In first case, a message copy from the source is transmitted to first $\mathrm{L}$ distinct nodes it finds after the message is generated. But in binary case, the source node transfers $\mathrm{L} / 2$ copies to the first $\mathrm{L} / 2$ nodes it encounters. These nodes then send half of the copies and continues this process until have only a single copy, which is directly sent to the destination.

\subsection{Spray-and-focus}

Unlike B-SNW routing, where in wait phase when a node has only a single copy which cannot be forwarded to any node except for waiting to direct transmission to destination but in focus phase of $\mathrm{SNF}$, this single copy can be sent to a more appropriate relay using single-copy utility-based routing scheme as follows: a node A keep a utility value for other node $\mathrm{B}$ in the simulation area and sends message if the utility of $\mathrm{A}$ is greater than the utility of $\mathrm{B}$ encountered to the destination plus the threshold value, which is an essential parameter [23-24].

\section{Simulation tools and set up}

Opportunistic Network Environment (ONE) simulator with program version of 1.5.1 is used in this paper. This section discusses ONE simulator with Graphical User Interface (GUI), and the environment modeling parameters.

\subsection{The one simulator}

ONE is basically designed for taking the analysis of routing techniques in ad hoc networks. Using ONE, user can get the understanding of mobility, contacts of node, routing, and handling of message which ensures a highly visualized reporting. The URL link and detail of the software is found in [25-26]. Source codes of ONE simulator are written using java programming language. Figure 2 shows the simulation area of the Helsinki city.

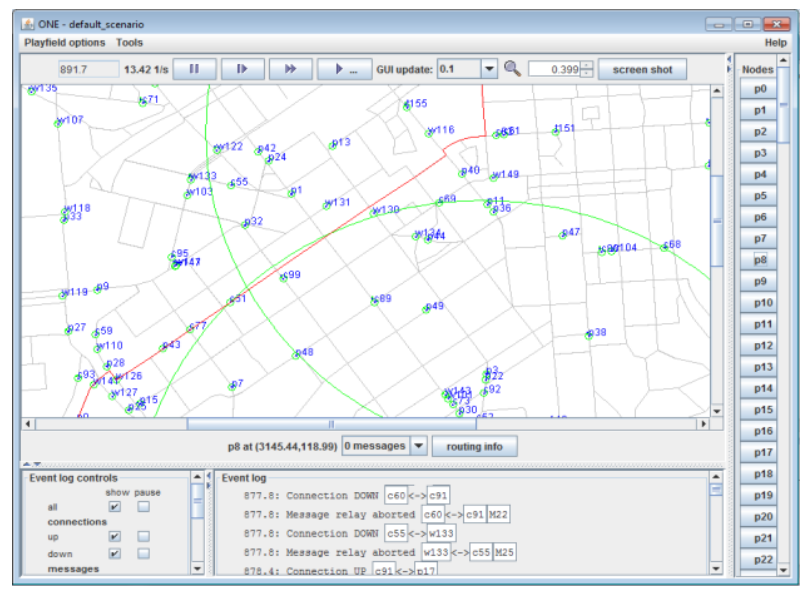

Fig. 2: Opportunistic Networks Scenario of Helsinki City Area

\subsection{Simulation environment setup}

Table I shows the simulation configuration for varying message TTL with constant buffer size of $5 \mathrm{MB}$, and varying buffer size with fixed TTL of 300 minutes, respectively. Table II summarizes the simulation configuration for routing algorithms.

Table 1: Simulation Environment Parameters

\begin{tabular}{ll}
\multicolumn{2}{c}{ Table 1: Simulation Environment Parameters } \\
\hline Parameters & Value \\
\hline Simulation Time & 24 hours \\
Update Interval & 1 second \\
Number of Nodes & 126 \\
Interface & Bluetooth Interface \\
Interface Type & Simple Broadcast Interface \\
Transmit Speed & $250 \mathrm{kbps}$ \\
Transmit Range & $10 \mathrm{~m}$ \\
Routing Protocols & Epidemic, PRoPHET, PRoPHETv2, MaxProp, \\
Buffer Size & RAPID, B-SNW, SNF \\
Message Genera- & 5MB, 10MB, 15MB, 20MB, 25 MB \\
tion Rate & 2, i.e., one message in 25-35 seconds \\
Message TTL & $50,100,150,200,250,300$ ( minutes) \\
Movement model & Shortest Path Map Based \\
Message Size & $500 \mathrm{~KB}-1 \mathrm{MB}$ \\
Simulation Area & $4500 \mathrm{~m} \times 3400 \mathrm{~m}$ \\
Size &
\end{tabular}


Table2: Parameters for Routing Algorithms

\begin{tabular}{lll}
\hline Routing Algorithm & Parameters & Value \\
\hline Epidemic & N/A & N/A \\
Prophet & Seconds In Time Unit & $30 \mathrm{~s}$ \\
Prophetv2 & Seconds In Time Unit & $30 \mathrm{~s}$ \\
Maxprop & Max. Size Of Probability & 50 \\
Rapid & Utility Algorithm & Average_Delay \\
B-Snw & No. Of Copies (L) & 6 \\
Snf & No. Of Copies (L) & 6 \\
\hline
\end{tabular}

\section{Simulation result and discussion}

This section discuses obtained results by running the simulations on the following performance metrics, i.e., delivery, latency, and overhead.

\subsection{Performance analysis on delivery probability}

Delivery probability is the sum of messages delivered to the destination over the sum of messages created at the source. With increasing buffer size, new message copies can be stored within a node. Hence, nodes can forward the copies through the next available nodes to the destination easily and so delivery probability increases. Due to the internal mechanism of SNF routing, it achieves higher delivery of message and that's why shows the better performance than other DTN routings with increasing buffer size as shown in Figure 3. On the other hand, as shown in Figure 4 , delivery probability increases with increase of TTL, i.e., lifetime of a packet for MaxProp, RAPID, B-SNW, and SNF but decreases for Epidemic, PRoPHET, and PRoPHETv2 when the number of nodes is greater than 100 . The reason of decreasing the delivery probability is that they (i.e., Epidemic, PRoPHET) do not apply any strategy of limiting the number of copies which could make the network congested. In both cases (Figures 3 and 4), SNF routing has higher delivery probability and so exhibits the better performance.

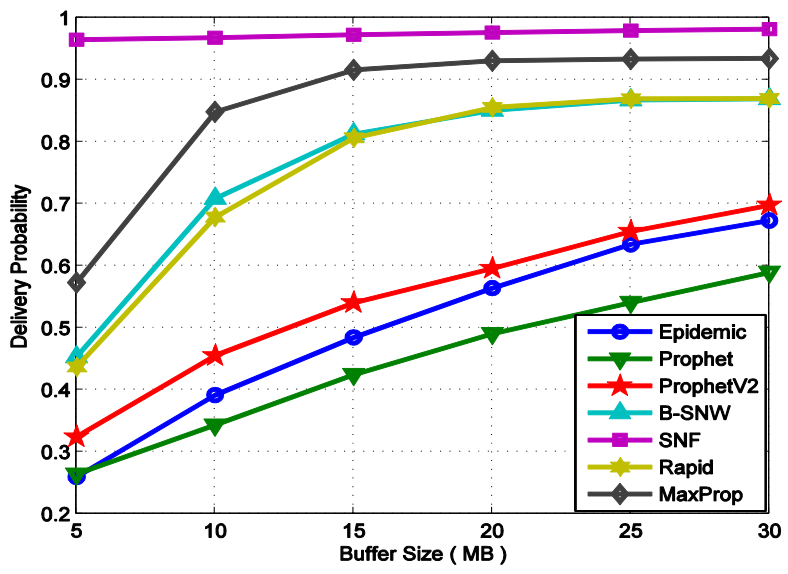

Fig. 3: Delivery Probability with Varying Buffer Size.

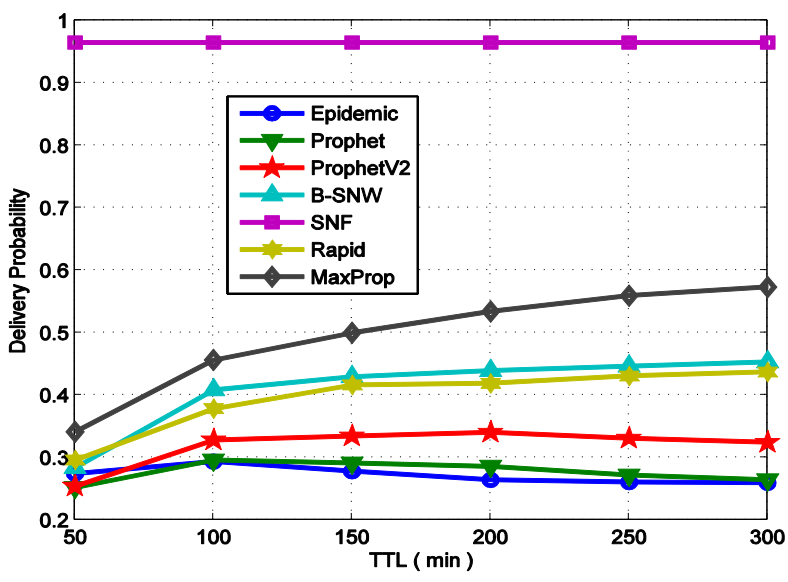

Fig. 4: Delivery Probability with Varying TTL.

\subsection{Performance analysis on average latency}

Average latency is the measure of average time between messages generated and messages received to destination. Average latency increases gradually in accordance with the increase in buffer size for all routing protocols except MaxProp as shown in Figure 5. Among all routings, SNF has the lowest average latency and that's why it shows the better performance than others. On the other hand, average latency increases with increase of TTL for all routing protocols except SNF which is constant (approximately zero). Hence, in both cases (Figures 5 and 6) SNF has very lowest average delay and so exhibits better performance.

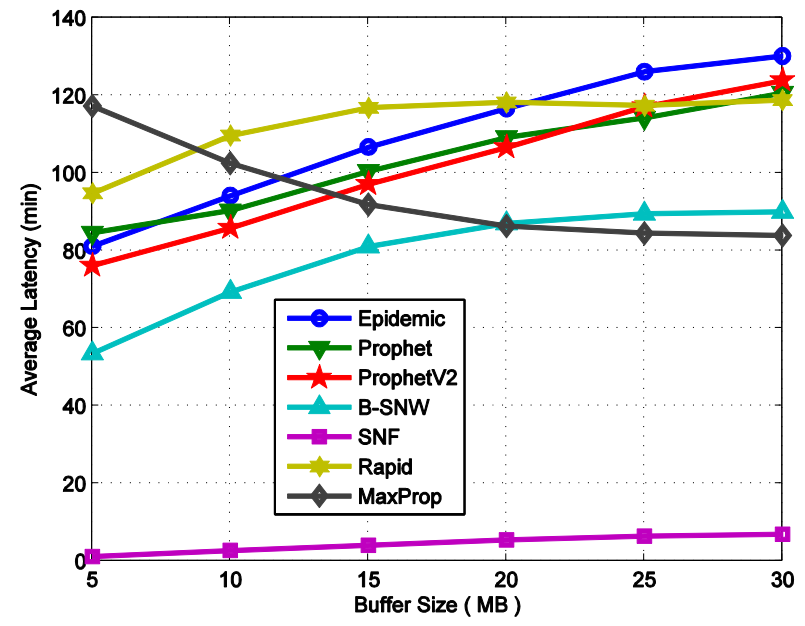

Fig. 5: Average Latency with Varying Buffer Size.

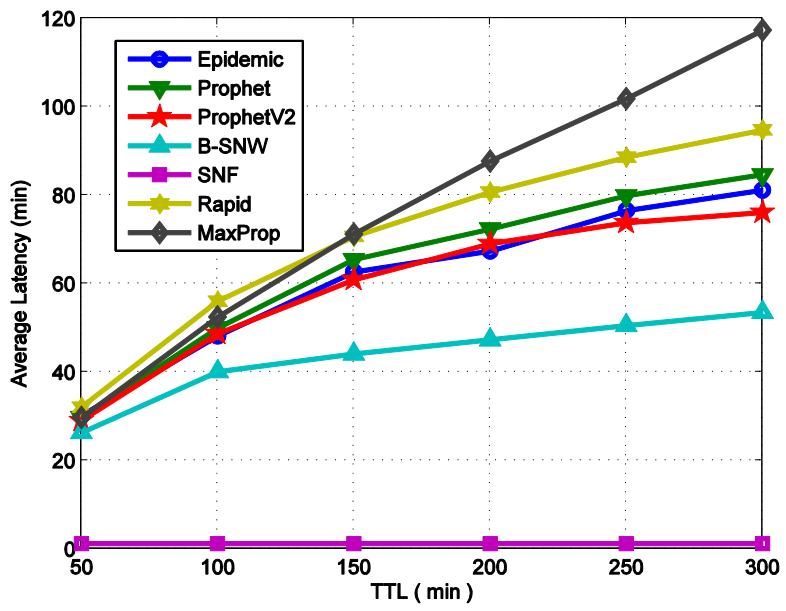

Fig. 6: Average Latency with Varying TTL.

\subsection{Performance analysis on overhead ratio}

Overhead ratio determines the number of redundant packets to replicate a packet successfully. It simply reflects the spent of message transfer in a network. With increase of buffer size, overhead ratio decreases for all routings except SNF which has approximately constant (zero) overhead. That's why SNF routing shows the better performance. Again, with increase of TTL, lowest overhead is provided by SNF routing. Therefore, in both cases as shown in Figure 7 and 8, SNF has the lowest overhead ratio and shows the better performance than other DTN routing protocols. 


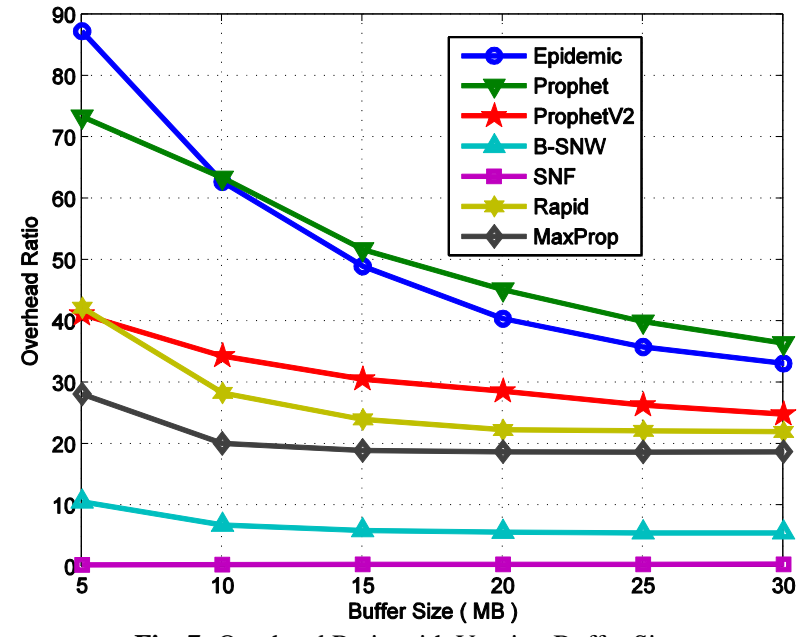

Fig. 7: Overhead Ratio with Varying Buffer Size.

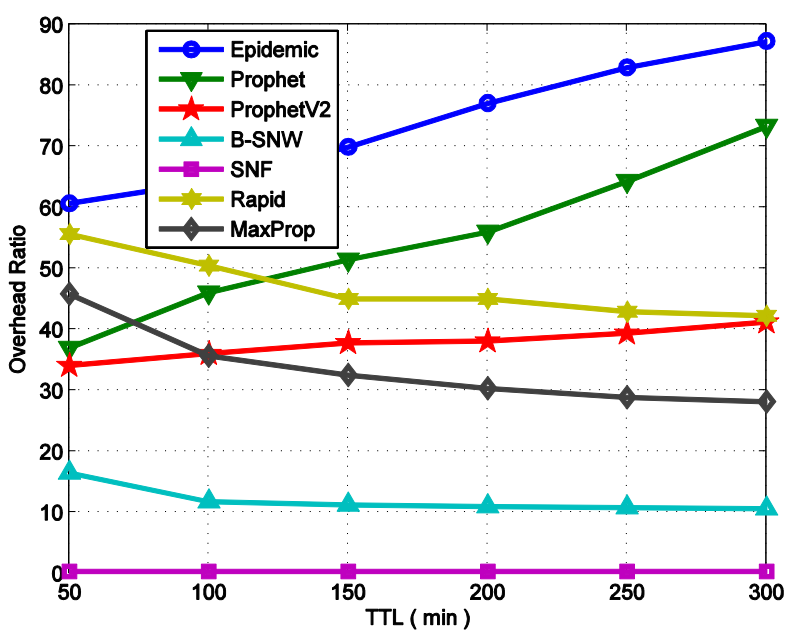

Fig. 8: Overhead Ratio with Varying TTL.

\section{Conclusion and future works}

In this research endeavor, the performance of seven replicationbased routings, i.e., Epidemic, PRoPHET, PRoPHETv2, Binary Spray-and-Wait , MaxProp, RAPID, and Spray-and-Focus (SNF) in an Intermittently Connected Mobile Networks (ICMNs) scenario has been investigated using ONE simulator. Investigation is done on following performance metrics, namely, delivery ratio, average delay and overhead ratio for changing buffer and Time-toLive (TTL), respectively. Simulation results demonstrated that SNF routing protocol exhibits the better performance with increase of buffer size and TTL in an ICMNs scenario because it has higher message delivery, lower delay and lower overhead than all the routing protocols considered here.

Further investigation can be done in future to investigate the above mentioned routing protocols in terms of energy consumptions and congestion control in the network.

\section{References}

[1] K. Fall, "A delay-tolerant network architecture for challenged internets," in Proc. of ACM SIGCOMM, Karlsruhe, Germany, Aug. 2003, pp.27-34. https://doi.org/10.1145/863955.863960.

[2] S. Jain, K. Fall, and R. Patra, "Routing in a delay-tolerant network," in Proc. of ACM SIGCOMM, Portland, USA, Oct. 2004, pp. 145 157. https://doi.org/10.1145/1015467.1015484.

[3] C. E. Perkins, and E. M. Royer, "Ad-hoc on-demand distance vector routing,"2nd IEEE Work. on Mob. Comp. Sys. and App., New Orleans, LA, USA, Feb. 1999, pp. 90-100. https://doi.org/10.1109/MCSA.1999.749281.
[4] D. B. Johnson, and D. A. Maltz, "Dynamic source routing in ad hoc wireless networks,"Mobile Com., Kluwer Academic Publishers, Feb. 1996, ch.5, pp. 153-181. https://doi.org/10.1007/978-0-585-29603-6_5.

[5] L. Pelusi, A. Passarella, and M. Conti, "Opportunistic networking: data forwarding in disconnected mobile ad hoc networks," IEEE Comm. Mag., vol. 44, no. 11, Nov. 2006, pp. 134-141. https://doi.org/10.1109/MCOM.2006.248176.

[6] Z. Zhang, "Routing in intermittently connected mobile ad hoc networks and delay tolerant networks: overview and challenges," IEEE Comm. Sur. and Tut., vol. 8, no. 1, Jan. 2006, pp. 24-37. https://doi.org/10.1109/COMST.2006.323440.

[7] J. Burgess, B. Gallagher, D. Jensen, and B. N. Levine, "Maxprop: routing for vehicle-based disruption-tolerant networks," in Proc. of IEEE INFOCOM, Barcelona, Spain, Apr. 2006, pp. 1-11. https://doi.org/10.1109/INFOCOM.2006.228.

[8] P. Juang, H. Oki, Y. Wang, M. Martonosi, L. S. Peh, and D. Rubenstein, "Energy-efficient computing for wildlife tracking: design tradeoffs and early experiences with zebranet," in Proc. of ACM ASPLOS, San Jose, CA, USA,Dec. 2002, pp. 96-107. https://doi.org/10.1145/605397.605408.

[9] A. Chaintreau, P. Hui, J. Crowcroft, C. Diot, R. Gass, and J. Scott, "Impact of human mobility on opportunistic forwarding algorithms," IEEE Tran. on Mob. Com., pp. 606-620, Jun. 2007. https://doi.org/10.1109/TMC.2007.1060.

[10] S. Burleigh, A. Hooke, L. Torgerson, K. Fall, V. Cerf, B. Durst, K. Scott, and H. Weiss, "Delay-Tolerant Networking: An approach to interplanetary internet," IEEE Comm. Mag, vol. 41, 2003, pp. 128-136. https://doi.org/10.1109/MCOM.2003.1204759.

[11] J. Partan, J. Kurose, and B. N. Levine, "A survey of practical issues in underwater networks," $1^{\text {st }}$ ACM Int. Works. on Underwater Net. in Con. with ACM MobiCom, Los Angeles, California, USA, Sep. 25 2006, pp. 17-24. https://doi.org/10.1145/1347364.1347372.

[12] G. E. Prescott, S. A. Smith, and K. Moe, "Real-time information system technology challenges for NASA's earth science enterprise," in Proc. of the 20th IEEE Real-Time Sys. Sym., Phoenix, AZ, USA, Dec. 1999.

[13] J.Ott, and D. Kutscher, "A disconnection-tolerant transport for drive-thru internet environments," in Proc. of IEEE INFOCOM, Miami, FL, USA, Mar. 2005, vol. 3, pp. 1849-1862. https://doi.org/10.1109/INFCOM.2005.1498464.

[14] A. Vahdat and D. Becker, "Epidemic routing for partially connected ad hoc networks," Tech Rep., Dept. of Computer Science, Duke University, CS-2000-06, Apr. 2000.

[15] A. Lindgren, A. Doria, and O. Scheln, "Probabilistic routing in intermittently connected networks," ACM Mob. Com. andComm. Rev., vol. 7, no. 3, pp. 19-20, Jul. 2003. https://doi.org/10.1145/961268.961272.

[16] S. Grasic, E. Davies, A. Lindgren, and A. Doria, "The evolution of a DTN routing protocol - PRoPHETv2," in Proc. of ACM SIGCOMM CHANTS, Las Vegas, Nevada, Sep. 2011, pp. 27-30. https://doi.org/10.1145/2030652.2030661.

[17] A. Balasubramanian, B. N. Levine, and A. Venkataramani, "DTN routing as a resource allocation problem," in Proc. of ACM SIGCOMM, Kyoto, Japan, Aug. 2007, pp. 373-384 https://doi.org/10.1145/1282380.1282422.

[18] T. Spyropoulos, K. Psounis, and C. S. Raghavendra, "Spray and wait: an efficient routing scheme for intermittently connected mobile networks," in Proc. of ACM WDTN, Philadelphia, USA Aug.2005, pp.252-259. https://doi.org/10.1145/1080139.1080143.

[19] T. Spyropoulos, K. Psounis, and C. S. Raghavendra, "Spray and focus: efficient mobility-assisted routing for heterogeneous and correlated mobility," in Proc. of IEEE PerCom, White Plains, NY USA, Mar. 2007, pp. 79-85. https://doi.org/10.1109/PERCOMW.2007.108.

[20] M. S. Hossen, M. T. Ahmed, and M. S. Rahim, "Effects of Buffer Size and Mobility Models on the Optimization of Number of Message Copies for Multi-Copy Routing Protocols in Scalable Delay-Tolerant Networks," in Proc. of IEEE ICISET, IIUC, Bangladesh, Oct 2016. https://doi.org/10.1109/ICISET.2016.7856502.

[21] M. S. Hossen, and M. S. Rahim, "Performance Evaluation of Replication-Based DTN Routing Protocols in Intermittently Connected Mobile Networks," in Proc. of IEEE ICEEE, RUET, Nov 2015. https://doi.org/10.1109/CEEE.2015.7428229.

[22] K. Massri, A. Vernata, and A. Vitaletti, "Routing protocols for delay tolerant networks: a quantitative evaluation," $7^{\text {th }} A C M$ Work. on Per. Moni. and Meas. of Heter. Wireless and Wired Net., Paphos, Cyprus Island, Oct. 2012, pp. 107-114. https://doi.org/10.1145/2387191.2387207. 
[23] T. Small and Z. Haas, "Resource and performance tradeoffs in delay-tolerant wireless networks," in Proc. of ACM WDTN, Philadelphia, PA, USA, Aug. 2005, pp. 260-267. https://doi.org/10.1145/1080139.1080144.

[24] A. Chaintreau, P. Hui, J. Crowcroft, C. Diot, R. Gass, and J. Scott, "Impact of human mobility on the design of opportunistic forwarding algorithms," in Proc. of IEEE INFOCOM, Barcelona, Spain, Apr. 2006.

https://doi.org/10.1109/INFOCOM.2006.172.

[25] A. Keränen, J. Ott, and T. Kärkkäinen, "The ONE simulator for DTN protocol evaluation," in Proc. of $2^{\text {nd }}$ Int. Conf. on Sim. Tools and Tech., Rome, Italy, Mar. 2009. https://doi.org/10.4108/ICST.SIMUTOOLS2009.5674.

[26] Project page of the ONE simulator. [Online]. Available: http://www.netlab.tkk.fi/tutkimus/dtn/theone [Accessed May 2018]. 